Casos Clínicos

Arch. Esp. Urol., 61, 7 (819-822), 2008

\section{TUMOR NEUROENDOCRINO EN EL RIÑÓN EN HERRADURA: RIESGO RELATIVO DE ASOCIACION DE DOS ENTIDADES RELACIONADAS}

Roger Boix Orri, Miguel Julián Mora Durban, Javier Sánchez Macías, José Ruiz Domínguez, Sergi Bernal Salguero, Joan Areal Calama y Josep Mํ Saladié Roig.

Servicio de Urología y Trasplante. Hospital Universitario Germans Trias i Pujol. Badalona. Barcelona. España.

Resumen.- OBJETIVO: El tumor neuroendocrino (TNE) renal primario es una neoplasia muy rara aunque con una mayor frecuencia de aparición en el riñón en herradura (RH). Desde que en 1966 Resnik publicó el primer caso, aproximadamente 2 de cada 10 nuevos diagnósticos aparecen en riñones con esta malformación. A partir del diagnóstico de un caso de TNE primario en RH, calculamos su riesgo relativo de presentación como entidades asociadas

Roger Boix i Orri

Alfons XII, $17-4^{\circ} 3^{\circ}$

08915 - Badalona. Barcelona. (España).

boixru@gmail.com

Trabajo recibido: 26 de noviembre 2007.
MÉTODOS: Varón de 63 años al que descubrimos incidentalmente una masa sólida de $8 \mathrm{~cm}$. ubicada en el istmo de un RH. En el momento del diagnóstico no se evidenciaron signos de extensión locoregional, sospecha de otra neoplasia primaria extrarrenal. El paciente fue sometido a heminefrectomía y el estudio histológico confirmó el diagnóstico de TNE.

Tras una revisión sistemática de la literatura utilizando múltiples plataformas (Blackwell, Ovid, Proquest, PubMed, Science Direct y Wileyl, el nuestro, representa el caso decimoprimero de TNE en RH de un total de 57 TNE originados en riñón.

RESULTADOS: Pese a su escasa frecuencia, un $19.3 \%$ de los TNE se presentan en un RH. Utilizando una incidencia de $R H$ en la población general de 1/400, el riesgo relativo calculado de TNE en $R H$ respecto al de un riñón normal es 77 veces mayor.

CONCLUSIONES: El riesgo relativo de presentación de un TNE en el RH es enormemente superior al de la población general. Este diagnóstico debe sospechase en pacientes con $R H$, sobre todo si el tumor asienta en el istmo renal. Dado su potencial curso clínico agresivo y pobre respuesta a otras terapias, las medidas encaminadas a mejorar el tratamiento quirúrgico deben ser consideradas prioritarias.

Palabras clave: Neoplasia renal. Tumor neuroendocrino. Tumor carcinoide renal. Anomalías renales. Riñón en herradura.

Summary.- OBJECTIVE: Primary renal neuroendocrine tumor (NET) is a very rare neoplasia with a higher frequency of appearance in horseshoe kidney (HK). From 1966, when Resnick published the first case, approximately 2 of each 10 new cases appear in kidneys with this malformation. From the diagnosis of a case of primary NET in a HK, we calculated the relative risk of their presentation as associated entities.

METHODS: 63-year-old male with the incidental diagnosis of an $8 \mathrm{~cm}$ solid renal mass in the isthmus of a HK. At the time of diagnosis there were no signs of local-regional extension of the primary neoplasia neither metastasis. The patient underwent nephrectomy and pathologic study confirmed the diagnosis of NET. After a systematic review using multiple search platforms (Blackwell, Ovid, Proquest, PubMed, Science Direct, and Wiley) our case is No. 11 of NET in HK from a total of 57 renal NET.

RESULTS: Despite its very low frequency, 19.3\% of NET present in HK. Using 1/400 as the incidence of HK in general population, the calculated relative risk in HK is 77 times greater than that of a normal kidney.

CONCLUSIONS: The relative risk of presentation of NET in $H K$ is enormously superior than that of general population. 
This diagnosis must be suspected in patients with HK, mainly if the tumor is located in the renal isthmus. Due to its potentially aggressive clinical course and poor response to other therapies, measures for improvement of surgical treatment must be considered priority.

Keywords: Renal neoplasia. Neuroendocrine tumor. Renal carcinoid tumor. Renal anomalies. Horseshoe kidney.

\section{INTRODUCCIÓN}

Los TNE son neoplasias que provienen del neuroectodermo embrionario, muy poco frecuentes. Pueden aparecer en cualquier tejido u órgano, incluso en aquellos que, como el riñón, no poseen normalmente células neuroendocrinas. Su localización más habitual es el aparato respiratorio o digestivo, siendo excepcionales en el sistema genitourinario, dónde pueden asentar en riñón, uretra, próstata y testículo (1). Dependiendo del grado histológico se dividen en: bien diferenciado (TNE carcinoide), carcinoma neuroendocrino, carcinoma neuroendocrino mal diferenciado de célula grande y carcinoma de célula pequeña. En general, los TNE de riñón se consideran neoplasias agresivas (2).

Desde que Resnick describe el primer caso (3), se han publicado series cortas y comunicaciones de casos aislados, hasta totalizar más de 50 TNE renales incluidos en una revisión publicada recientemente (4). Gran parte de los autores coinciden en señalar la asociación de esta neoplasia con el RH, que juntas, representan un importante reto terapéutico, ya que si bien la opción quirúrgica suele ser viable, concurren factores de complejidad técnica, mal pronóstico y pobre respuesta a los tratamientos complementarios.

Lo infrecuente de esta patología, ha determinado cifras dispares en cuanto a su incidencia, por lo que, una vez confirmado un nuevo caso y tras revisar la literatura, actualizamos el cálculo del riesgo relativo de la asociación TNE y RH.

\section{CASO CLÍNICO}

Varón de 63 años, sin antecedentes de interés, al que por ecografía abdominal solicitada para el estudio de un síndrome prostático, se le diagnosticó de $\mathrm{RH}$ con una masa sólida que asienta en el istmo renal. El TAC abdominal (Figural) confirmó la presencia de un tumor de $8 \mathrm{~cm}$. en el istmo renal sin evidenciar adenopatías, metástasis locoregionales ni lesiones a distancia en el estudio de extensión.

El estudio vascular preoperatorio mediante arteriografía, demostró la existencia de dos arterias renales con nacimiento ortotópico. Las imágenes selectivas de la arteria renal derecha, evidenciaron vasos neoformados a expensas de la rama medial en su bifurcación con normalidad del árbol venoso intra y extrarrenal (Figura 2).

Con la presunción diagnóstica de carcinoma renal, se practicó istmectomía y nefrectomía izquierda a través de una laparotomía media. Durante el acto operatorio se apreció que la pelvis renal y el uréter izquierdos se hallaban infiltrados por el tumor. La anatomía patoló-

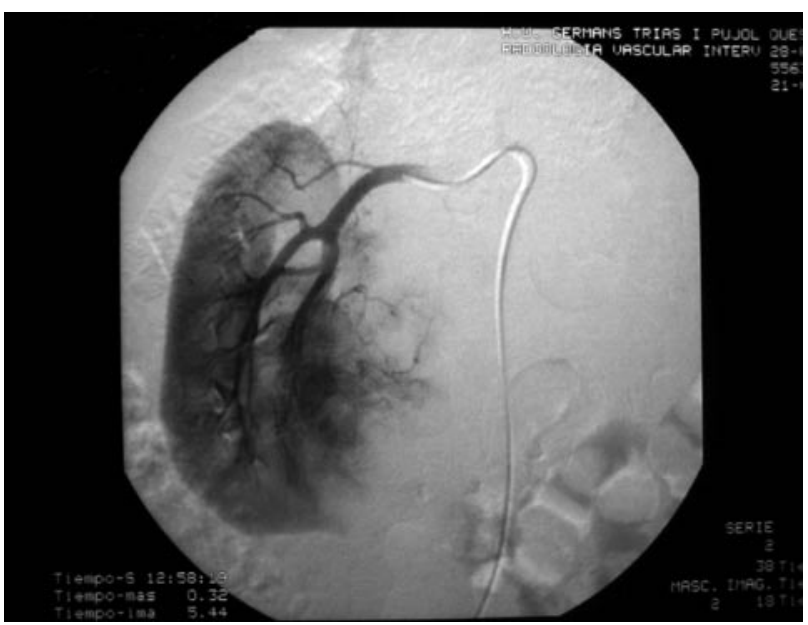

FIGURA 2. Detalle de arteriografía selectiva renal

derecha. Se observan vasos neoformados aberrantes en el seno de la lesión.
FIGURA 1. TAC abdominal con contraste en fase de elimi- nación. Se observa tumoración de unos $8 \mathrm{~cm}$. asentando
en istmo renal de un riñón en herradura.

nación. Se observa tumoración de unos $8 \mathrm{~cm}$. as
en istmo renal de un riñón en herradura.

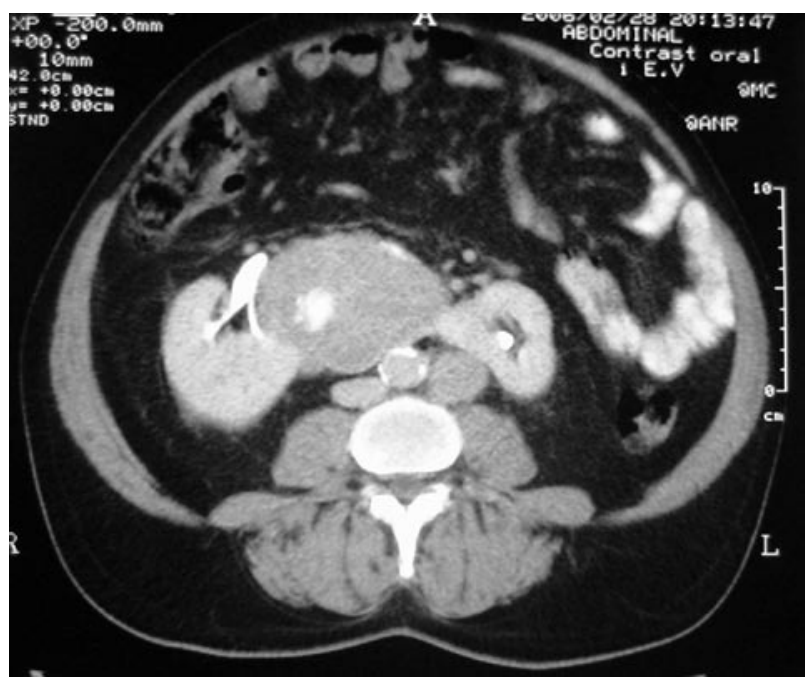


gica mostró una lesión renal neoformada de $10 \mathrm{~cm}$. afectando cápsula, grasa perirrenal, así como, la vía urinaria izquierda. La histología fue de carcinoma neuroendocrino de célula no pequeña. El estudio inmunohistoquímico resultó positivo para CD-56 y sinaptofisina (Figura 3).

A los 4 meses de la intervención, el TAC abdominal apreció un nódulo sólido de $2 \mathrm{~cm}$. en el polo inferior del riñón derecho, así como, adenopatías para-aórticas izquierdas y múltiples nódulos retrocrurales subcentimétricos. El estudio PET-TC mostró que las lesiones descritas eran sugestivas de metástasis. El paciente fue sometido a tratamiento con radioterapia externa (RT) y quimioterapia (QT) (carboplatino y VP-16) con buena tolerancia y respuesta parcial. El paciente permaneció asintomático y sin efectos secundarios tardíos de RT ni QT. Dos años tras la cirugía el TAC abdominal muestra la persistencia de pequeños ganglios retroperitoneales, sin otros hallazgos destacables.

\section{DISCUSIÓN}

El RH es la anomalía congénita de fusión renal más frecuente. Su incidencia se estima del $0.25 \%$ en la población general (5). Estos riñones presentan un riesgo más elevado de malignización con el desarrollo de neoplasias que suelen ser carcinomas de células renales (CCR) aunque también se han descrito tumores de células transicionales, tumor de Wilms, linfomas, carcinomas escamosos, TNE y sarcomas (6).

La incidencia de CCR en los $\mathrm{RH}$, es similar respecto a los riñones no fusionados, en cambio, hay una mayor incidencia de tumor de Wilms y carcinomas de células transicionales, en los pacientes con $\mathrm{RH}$, estimándose un riesgo relativo para estas patologías de 3 a 4 veces superior que en la población general (7). Aunque el riego de aparición de TNE en el RH se ha descrito como mucho mayor que la de otros tumores, la rareza del TNE ha ocultado la importancia de dicha asociación $(2,8,9)$.

La mayoría de autores coinciden en señalar la asociación de esta neoplasia con el $\mathrm{RH}$, estableciéndose varias teorías para explicar la coincidencia de ambas patologías. El hecho que el istmo renal se encuentre habitualmente afecto y la hipótesis de un origen teratogénico del mismo, podrían explicar su asociación y comportamiento.

La literatura reciente ofrece datos dispares en cuanto al riesgo relativo de TNE en el RH, según son descritos. Utilizando la misma fórmula que utilizaron por primera vez Krishnan y cols. (5) y que adoptaron artículos posteriores, calculamos el nuevo riesgo relativo, considerando que la incidencia de $\mathrm{RH}$ en la población general es de 1/400. Incluyendo nuestro caso, hemos contabilizado un total de 57 TNE renales, de los que 11 asientan en $\mathrm{RH}$, lo que supone un $19.3 \%$ del total.

Usando los datos de la última revisión de Romero y cols. (2), si aceptamos que se notifican por igual tanto los TNE en riñones normales como en $\mathrm{RH}$, obtenemos que el riesgo relativo aproximado de TNE en $\mathrm{RH}$ es 77 veces superior.

Para explicar el origen de los TNE renales se han postulado varias hipótesis. La primera se basa en la presencia de células neuroendocrinas halladas en el parénquima renal, de la misma forma que se encuentran por en el
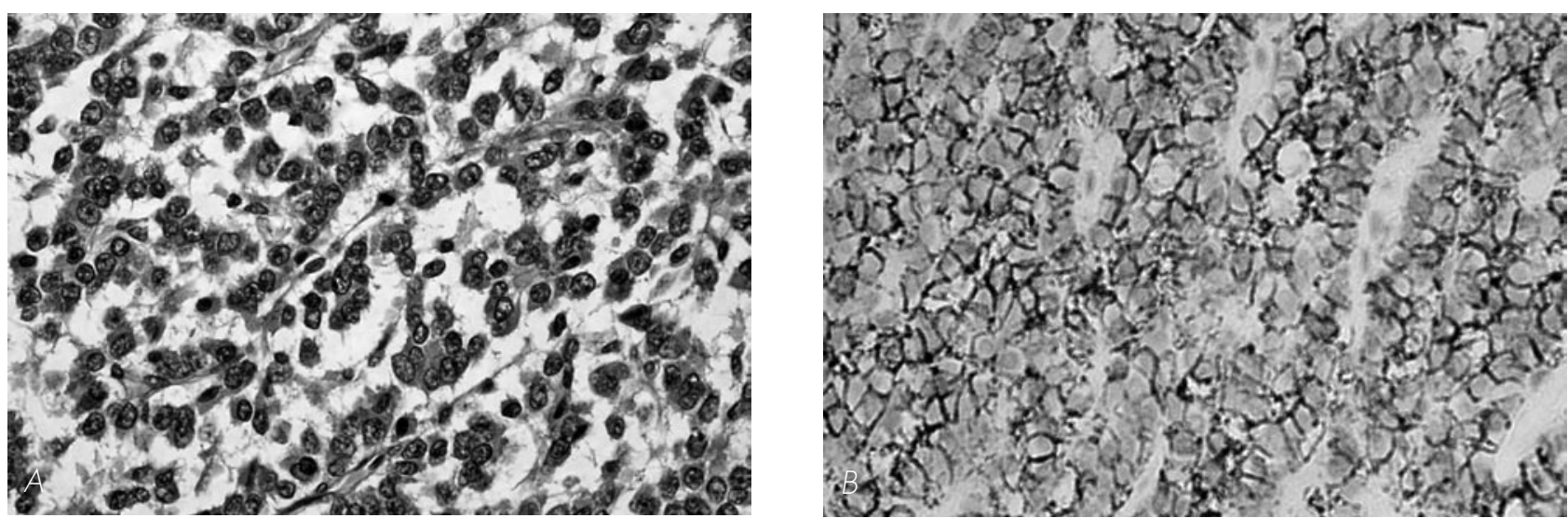

\section{FIGURA 3. Histología}

A) Hematoxilina-eosina: Proliferación de células epiteliales dispuestas en patrón organoide y en nidos, separados por una fina trama vascular. Células de tamaño medio con relación núcleo citoplasma invertida. Núcleo ovalado con cromatina grosera y nucleolo.

B) Inmunohistoquímica: Positividad para marcadores neuroendocrinos (CD56, Sinaptofisina). 
resto del aparato urinario $(3,10)$. La segunda se basa en la diferenciación de células primitivas hacia células neuroendocrinas (2). La tercera plantea la posibilidad de la presencia de células neuroendocrinas asociadas con la metaplasia intestinal de las células de la pelvis renal; esta se produciría como reacción a infecciones, litiasis u otros fenómenos lesivos (10). La cuarta hipótesis se fundamenta en el hecho que las células neuroendocrinas renales se encuentran entre epitelio teratógeno respiratorio o intestinal en el riñón. Esta última teoría es importante en nuestro caso, ya que para el origen del istmo del $\mathrm{RH}$ también se postula un origen teratogénico $(2,8)$.

El diagnóstico de sospecha del TNE es difícil. Normalmente aparecen en pacientes asintomáticos o con síntomas poco específicos, como fiebre y dolor abdominal entre otros. El síndrome carcinoide sólo está presente en un $10-15 \%$ de los casos, mientras que otros síndromes secundarios a la liberación de hormonas como los de Zollinger-Ellison, Verner-Morrison o Cushing, también son poco habituales. $(4,6)$. El diagnóstico preoperatorio se basa en la demostración de sustancias neuroendocrinas en orina o sangre, siendo la detección de 5-HIAA (metabolito de la serotonina) en orina, el más usado. Hasta la fecha, no existe ningún test que pueda filiar una masa renal como un TNM silente y la gammagrafía con octreotide sólo resulta útil para tumores con actividad. (1).

En tumores localizados, el tratamiento quirúrgico agresivo es el idóneo, ya que QT y RT son sólo paliativas (1, 6). En este sentido y para planear la estrategia operatoria, un estudio vascular del RH mediante angio-TAC o arteriografía, puede ser de gran ayuda. Finalmente, el factor pronóstico más importante de estas neoplasias es el estadio tumoral. Llama la atención que, a diferencia con nuestro caso, los TNE surgidos en RH se describen con evolución más favorable y mayor supervivencia, incluso aquellos con enfermedad diseminada compararlos con los que asientan en riñones normales $(2,9)$.

\section{CONCLUSIONES}

El riesgo relativo de presentación de TNE en el RH es enormemente superior al de la población general: 77 veces mayor. El TNE debe ser sospecha diagnostica en estos pacientes, sobre todo si el tumor asienta en el istmo renal. Por su potencial curso clínico agresivo y mala respuesta a otras terapias, las medidas encaminadas a mejorar el tratamiento quirúrgico deben ser consideradas prioritarias.

\section{BIBLIOGRAFÍA y LECTURAS RECOMENDADAS ( ${ }^{*}$ lectura de interés $y$ ** lectura fundamental)}

**1. MURALI, R.; KNEALE, K.; LALAK, N. y cols.: "Carcinoid Tumors of the Urinary Tract and Prostate". Arch. Pathol. Lab. Med., 130: 1693, 2006.

**2. MOTTA, L.; CANDIANO, G.; PEPE, P. y cols.: "Neuroendocrine tumor in a horseshoe kidney. Case report and updated follow-up of cases reported in the literature". Uro. Int., 73: 361, 2004.

3. RESNIK, M.E.; UNTERBERGER, H.; McLOUGHLIN, P.T.: "Renal carcinoid producing the carcinoid syndrome". Med. Times, 94: 895, 1966.

*4. ROMERO, F.; RAIS-BAHRAMI, S.; PERMPONGKOSOL, L. y cols.: "Primary Carcinoid tumors of the Kidney". J. Urol., 176: 2359, 2006.

5. KRISHNAN, B.; TRUONG, L.; SALEH, G. y cols.: "Horseshoe Kidney is associated with an increased relative risk of primary renal carcinoid tumor". J. Urol., 157: 2059, 1997.

*6. LANE, B.; CHERY, F.; JOUR, G.; y cols.: "Renal neuroendocrine tumors: A clinicopathological study". BJU Int., 100: 1030, 2007.

7. MESROBIAN, H.G.; KELALIS, P.; HRABOVASKY, E. y cols.: "Wilms tumor in horseshoe kidneys: A report from the National Wilms Tumor Study". J. Urol., 133: 1002, 1985.

8. RODRÍGUEZ, F.; GÓMEZ, X.; VALERIO, L. y cols.: "Carcinoid tumor arising in a horseshoe kidney". Int. Urol. Nephrol., 39: 373, 2006.

9. MUFARRIJ, P.; VARKARAKIS, I.M.; STUDEMAN, L. y cols.: "Primary renal carcinoid tumor with liver metastases detected with somatostatin receptor imaging". Urology, 65: 1002, 2005.

10. MACHET, M.C.; STEPHAVOV, E.; DE MURET, A. y cols.: "Primary carcinoid tumor of the kidney associated with cystic malformation of the kidney". Ann. Pathol., 14: 410, 1994. 